
\title{
28 Research Square \\ Prediction of Blood Pressure Change During Surgical Incision Under Opioid Analgesia Using a New Opioid Sensitivity Index: A Cross-Sectional Study
}

\section{Satoshi Kamiya}

Department of Anesthesiology and Critical Care, Hiroshima University

Ryuji Nakamura ( $\nabla$ r-nacamura@hiroshima-u.ac.jp)

Department of Anesthesiology and Critical Care, Hiroshima University

Noboru Saeki

Department of Anesthesiology and Critical Care, Hiroshima University

Takashi Kondo

Department of Anesthesiology and Critical Care, Hiroshima University Hirotsugu Miyoshi

Department of Anesthesiology and Critical Care, Hiroshima University

\section{Soushi Narasaki}

Department of Anesthesiology and Critical Care, Hiroshima University

\section{Atsushi Morio}

Department of Anesthesiology and Critical Care, Hiroshima University

\section{Masashi Kawamoto}

Medical Corporation JR Hiroshima Hospital

\section{Harutoyo Hirano}

Academic Institute, College of Engineering, Shizuoka University

\section{Toshio Tsuji}

Graduate School of Advanced Science and Engineering, Hiroshima University

\section{Yasuo Tsutsumi}

Department of Anesthesiology and Critical Care, Hiroshima University

\section{Research Article}

Keywords: The International Association, Opioid inhibition, sympathetic response, Nociceptive stimuli

Posted Date: January 22nd, 2021

DOI: https://doi.org/10.21203/rs.3.rs-149561/v1 
License: (c) (i) This work is licensed under a Creative Commons Attribution 4.0 International License. Read Full License 


\section{Abstract}

Opioid inhibition of nociceptive stimuli varies in individuals and is difficult to titrate. We have reported the vascular stiffness value $(K)$ as a standard monitor to quantify sympathetic response with high accuracy. On the contrary, among individuals, a considerable variation in the rate of change in $\mathrm{K}$ for constant pain has been observed. In this study, we proposed a new index, minimum evoked current of $\mathrm{K}\left(\mathrm{MEC}_{\mathrm{K}}\right)$ and evaluated its accuracy in predicting sympathetic response to nociceptive stimuli under constant opioid administration. Thirty patients undergoing open surgery under general anesthesia were included. After anesthetic induction, remifentanil was administered at a constant concentration of $2 \mathrm{ng} / \mathrm{ml}$ at the effect site followed by tetanus stimulation. $\mathrm{MEC}_{\mathrm{K}}$ was defined as the minimal current needed to produce a change in $\mathrm{K} \mathrm{MEC}_{\mathrm{K}}$ significantly $(\mathrm{P}<0.001)$ correlated with the rate of change of systolic blood pressure during skin incision $\left(\mathrm{ROC}_{\mathrm{BP}}\right)$. Bland-Altman plot analysis using the predicted $\mathrm{ROC}_{\mathrm{BP}}$ calculated from $\mathrm{MEC}_{\mathrm{K}}$ and the measured $\mathrm{ROC}_{\mathrm{BP}}$ showed that the prediction equation for $\mathrm{ROC}_{\mathrm{BP}}$ was highly accurate. This study showed the potential of $\mathrm{MEC}_{\mathrm{K}}$ to predict blood pressure change during surgical incision under opioid analgesia.

\section{Introduction}

The International Association for the Study of Pain defines pain as an aversive sensory and emotional experience. Patients under general anesthesia are unconscious and do not have an aversive experience. However, anesthesiologists commonly use analgesics in addition to sedatives because nociceptive stimuli can cause noxious autonomic reflexes. Opioids are the most commonly used analgesics during general anesthesia due to their lack of a ceiling effect. Opioids exert their analgesic effects mainly by inhibiting sensory nerve transmission in the spinal cord and by inhibiting the excitation of pain conduction pathways in the brain. When opioids are administered during general anesthesia, noxious autonomic reflexes are suppressed and multiple parameters such as heart rate, blood pressure, electrocardiogram, and respiratory rate are affected. During surgery, anesthesiologists rely on these parameters to estimate pain levels and adjust the opioid dosage. However, this nociceptive stimuliinduced sympathetic response varies among individuals, and therefore, so do individual anesthetic requirements. Adverse events, either as an unexpected increase in blood pressure due to underdosing or delayed arousal due to overdosing, are common. Thus, if the individual's opioid requirement can be accurately quantified in advance, more stable general anesthesia can be performed.

Nociceptive stimuli input to the central nervous system is output to effector organs such as the heart and blood vessels via the sympathetic nervous system. Opioids inhibit the input of nociceptive stimuli to the central nervous system. Therefore, the administration of opioids blunts the sympathetic response to nociceptive stimuli. In other words, by accurately quantifying the sympathetic response to a given nociceptive stimulus under opioid administration, we can determine the relationship between the opioid dose and the response to the nociceptive stimulus and quantify opioid sensitivity. Photoplethysmography (PPG), an increasingly popular tool, has recently begun to be used to measure sympathetic cutaneous 
blood flow responses for quantifying sympathetic nerve activity in peripheral vasculature [1-4]. However, PPG measures blood flow at the measurement site and does not, in principle, directly indicate the degree of sympathetic response. Therefore, we proposed a method to extract only the degree of vasoconstriction from sympathetic cutaneous blood flow responses to PPG and quantify it as vascular stiffness value (K) [5]. We reported that $K$ could be used to quantify pain and reflect the activity of the sympathetic nervous system $[6,7]$. Moreover, we reported that $K$ value reflects changes in pain caused by the administration of opioids during general anesthesia and that the response of $\mathrm{K}$ to pain was attenuated with increasing doses of opioids [8-10].

On the contrary, among individuals, a considerable variation in the rate of change in $\mathrm{K}$ for constant pain has been observed $[9,10]$. We speculated that this may have been due to the fact that the pathway of nociceptive stimuli, which travel from the peripheral nerves through the central nervous system to effector organs such as peripheral blood vessels, is strongly influenced by autonomic changes due to aging and coexisting diseases. While opioids inhibit the afferent pathway of pain perception, the rate of change of $\mathrm{K}$ represents the intensity of the sympathetic response or the efferent pathway. In other words, the rate of change in $\mathrm{K}$ includes information from both efferent and afferent pathways, so the measurement results may vary depending on the sensitivity of the effector. Therefore, we hypothesized that the "intensity of nociceptive stimuli" at which sympathetic responses appear is a better indicator of opioid sensitivity than the "intensity of sympathetic responses" to nociceptive stimuli (that is, the rate of change in K) because it exclusively extracts only information regarding afferent pathways.

In the present study, we measured two indices of opioid sensitivity: the minimum stimulus intensity that elicits a change in $\mathrm{K}$ and the rate of change in $\mathrm{K}$ for specific stimuli. We then assessed which index predicted the rate of change in blood pressure $\left(\mathrm{ROC}_{\mathrm{BP}}\right)$ more accurately during a skin incision under a constant effect-site concentration of opioids.

\section{Patients And Methods}

\section{Patients}

Prior to the study, we received approval from the Ethics Committee of Hiroshima University ('Hi'-226, 'E'-1523-1) and registered the clinical trial (registry: university hospital medical information network, registration number: UMIN000041816, principal investigator's name: Satoshi Kamiya, Date of registration: July 9th, 2019). This study was conducted in accordance with the Declaration of Helsinki and STROBE statement. The study population consisted of patients over 20 years of age who underwent open surgery under general anesthesia from July 2019 to October 2019. A total of 30 patients gave their written informed consent before the study. All procedures were conducted at Hiroshima University Hospital. We excluded patients with irregular R-R via electrocardiogram (ECG), inability to perform invasive arterial pressure measurements in the radial artery, significant hemodynamic or neurological impairment in the upper extremity, and severe stenosis or occlusive lesions in the coronary arteries or cerebral vessels. 
Before induction of anesthesia, a photoplethysmography probe (TL-271T, Nihon Kohden, Tokyo, Japan) on the middle finger of the left hand, ECG on the chest, electroencephalogram (EEG; Entropy, GE Healthcare UK Ltd., Buckinghamshire, UK) on the anterior forehead, neuromuscular blockade monitoring device (NMT- Neuromuscular Transmission, GE Healthcare UK Ltd., Buckinghamshire, UK) on the ulnar side of the forearm of the right hand, and a non-invasive blood pressure cuff on the right upper arm were placed. In all patients, a preoperative dosing plan was developed to achieve a predicted effect-site concentration of $2 \mathrm{ng} / \mathrm{ml}$ remifentanil. Minto's pharmacokinetic model [11] was used to calculate predicted effect-site concentrations. Administration of remifentanil was initiated as per the dosing plan, and propofol $3 \mu \mathrm{g} / \mathrm{mL}$ was used to induce anesthesia using a target-controlled infusion (TCl) pump with built-in 'Diprifusor' (TE-371, Terumo, Tokyo, Japan). After the patient was unconscious, $50 \mathrm{mg}$ of rocuronium was administered and a $22 \mathrm{G}$ catheter was secured in the left radial artery for measuring arterial blood pressure (ABP). Data from ECG, ABP, and PPG were output to a personal computer from a bedside patient monitor (BSS-9800, Nihon Kohden, Tokyo, Japan) and were used to calculate $\mathrm{K}$ values in real-time.

After the predicted effect-site concentration of remifentanil reached a steady state at $2 \mathrm{ng} / \mathrm{ml}$, tetanus stimuli at $50 \mathrm{~Hz}$ for $5 \mathrm{~s}$ were delivered through a two-pole body surface electrode on the ulnar side of the right hand using the INNERVATOR 252 (Fisher \& Paykel Healthcare, Auckland, New Zealand). The current value was initially $10 \mathrm{~mA}$ and increased in increments of $10 \mathrm{~mA}$ until $80 \mathrm{~mA}$, the maximum output of the INNERVATOR 252, was reached. Thus, a total of eight tetanus stimulation sessions were performed. Once the $\mathrm{K}$ value returned to its pre-stimulus value, the next tetanus stimulation was performed. Subsequently, tracheal intubation was performed using a Macintosh laryngoscope or McGRATH ${ }^{\mathrm{TM}}$ MAC video laryngoscope (Medtronic, Dublin, Ireland). All endotracheal intubations were successfully executed in a single attempt. Thereafter, the same amount of propofol TCl target blood levels and rate of remifentanil administration were maintained until the skin incision was performed.

\section{Calculation of $K$}

In this study, the motion of the vessel wall was predicted using a model in which springs and dampers were aligned in parallel to the diameter direction of the vessel. The arterial wall, moved by a force (blood pressure) applied in the direction of the diameter of the vessel, was dampened by springs and dampers that represent the stiffness and viscosity of the vessel wall itself. The behavior of the vessel wall after damping was observed as a change in the PPG waveform (Figure 1). We used the spring constant in this model equal to $K$. Details of the calculation of $K$ value have been reported previously [8]. In brief, we first detected the heartbeats with the R wave of the ECG and cut out the ABP and PPG waveforms for each heartbeat. All wave height data associated with one heartbeat were provided in the following equations:

$\mathrm{dPb}(t)=\operatorname{KdPI}(t)+\operatorname{BdPI}(t),(1)$ 
$\mathrm{dPb}(t)=\mathrm{Pb}(t)-\mathrm{Pb}\left(t_{0}\right), \mathrm{dPI}(t)=\mathrm{PI}(t)-\mathrm{PI}\left(t_{0}\right)$

$\mathrm{dPI}(t)=\mathrm{PI}(t)-\mathrm{PI}\left(t_{0}\right),(3)$

The time at which the change was first observed was considered $t_{0}$, ABP wave height at time $t$ was $\mathrm{Pb}(t)$, and PPG wave height at time $t$ was represented as $\mathrm{PI}(t)$. $\mathrm{PI}(t)$ is equal to the first derivative of the PPG wave height. The coefficients for vascular stiffness and vascular viscosity values, $K$ and $B$, respectively, were determined as one value per heartbeat by performing a least-squares fit of the equation to all data acquired in each heartbeat. $\mathrm{K}$ determined in this way was actually displayed automatically at the bedside in real-time, every heartbeat. If the coefficient of determination was less than 0.95 , or if $\mathrm{K}$ and/or $\mathrm{B}$ were negative, the data were removed from the analysis. Only $\mathrm{K}$ was analyzed in the present study because previous studies have estimated that $\mathrm{K}$ was a promising candidate as a measure of nociceptive stimulus intensity [8].

\section{Statistical analysis}

Figure 2 shows an example of how the MEC was measured in this study. Each parameter measurement in tetanus stimulation is described in the following text. First, pre-stimulus values were set as the median value determined throughout the $10 \mathrm{~s}$ that preceded tetanus stimulation. Post-stimulus values were the maximum values of $\mathrm{K}$, heart rate, systolic blood pressure, and minimum values of PPG amplitude for $20 \mathrm{~s}$ after tetanus stimulation. The post-stimulus values were divided by the pre-stimulus values and the percentage change in parameters in response to tetanus stimulation at each stimulus intensity was calculated. MEC was defined as the minimum stimulus intensity value that produced a change of more than $5 \%$ in the parameter during tetanus stimulation. However, once the change was greater than $5 \%$, and if the rate of change was less than $5 \%$ at a subsequent stage, the MEC calculated at a lower current value was rejected as noise. In other words, the MEC would eventually be one step stronger than the maximum stimulus intensity at each stimulus intensity, where the rate of change of the parameter was below $5 \%$. If no more than $5 \%$ change appeared at $80 \mathrm{~mA}$ tetanus stimulation, MEC was classified as outside the measurement range. The MEC for $\mathrm{K}, \mathrm{HR}, \mathrm{BP}$, and PPG were designated as $\mathrm{MEC}_{\mathrm{K}}, \mathrm{MEC}_{\mathrm{HR}}, \mathrm{MEC}_{\mathrm{BP}}$, and $M C_{P P G}$, respectively. The rate of change in $K$ before and after $80 \mathrm{~mA}$ tetanus stimulation $\left(\mathrm{K}_{\mathrm{R} 80}\right)$ was calculated as a measure of the rate of change for constant intensity nociceptive stimuli. The median systolic blood pressure of $10 \mathrm{~s}$ before the scalpel skin incision was defined as pre-BP, and the maximum systolic blood pressure between the skin incision and the start of electrocautery use was defined as post$\mathrm{BP}$. The $\mathrm{ROC}_{\mathrm{BP}}$ before and after skin incision was calculated by dividing post-BP by pre-BP. A first-order regression was performed to confirm the relationship between $M E C$ and $\mathrm{ROC}_{\mathrm{BP}}$. Specifically, a first-order regression equation was created between cases where $\mathrm{MEC}$ and $\mathrm{ROC}_{\mathrm{BP}}$ could be measured. A SmirnovGrubbs test was performed using this regression formula, and a measurement in which $\mathrm{P}$ was greater than or equal to 0.05 was removed as an outlier. The regression equation was remade for each of the removed outliers. The regression equation at the time outliers were removed was adopted as the final correlation line. Pearson's correlation coefficients were calculated using measurements at the time at which outliers were eliminated. The significance level was set at 0.05 . 
Further, we attempted to predict the $\mathrm{ROC}_{\mathrm{BP}}$ using $\mathrm{MEC}_{\mathrm{K}}$ and $\mathrm{K}_{\mathrm{R} 80}$. Specifically, the prediction equation was based on the first-order approximate equation previously developed, and the predicted $\mathrm{ROC}_{\mathrm{BP}}$ was calculated from $\mathrm{MEC}_{\mathrm{K}}$. For cases in which $\mathrm{MEC}_{\mathrm{K}}$ was outside the measured range, we fitted the mean value of $\mathrm{ROC}_{\mathrm{BP}}$ in out-of-range cases to the approximate equation and substituted the estimated value. $A$ similar first-order approximation and outlier treatment was performed for $\mathrm{K}_{\mathrm{R} 80}$ and $\mathrm{ROC}_{\mathrm{BP}}$. From the final approximate equation, the predicted $\mathrm{ROC}_{\mathrm{BP}}$ was calculated. A Bland-Altman analysis was performed for both measured and predicted $\mathrm{ROC}_{\mathrm{BP}}$, and fixed bias and limit of agreement range were calculated for each.

No prior studies are available on $\mathrm{MEC}_{\mathrm{K}}$, and therefore, the prior number of cases cannot be determined. Genetic polymorphisms related to opioid sensitivity were measured in the subjects at the same time the data used in this study were collected. In the genetic polymorphism study, the power test calculated the required number of cases as 30 , and the present study was conducted in 30 cases with the analysis of genetic polymorphisms. Moreover, the effect size was calculated to ensure a sufficient sample size. In Pearson's correlation analysis, the r-value, which is the correlation coefficient, was used as a measure of the effect size. All the r-values of the main results of this study indicate moderate-to-high effect sizes.

\section{Results}

A total of 30 patients (male, 15) were included in the study and the mean age of participants was 62 years. Thirteen patients underwent an upper abdominal incision, and 17 underwent a lower abdominal incision. None of the patients met the exclusion criteria. A summary of patient characteristics is shown in Table 1. ROC $\mathrm{BP}_{\mathrm{BP}}$ was $18.3 \pm 11.8 \%$ (mean $\pm \mathrm{SD}$ ).

Figure 3 shows the values of $K, H R, s B P$, and PPG amplitude after tetanus stimulation of each intensity. $K$ and SBP values tended to increase with the intensity of the stimulus current. PPG amplitude tended to decrease with increasing stimulus intensity. HR did not show consistent changes. Even for K, which produced the least individual differences, actual measured values had a large degree of individual variability. Therefore, it was not possible to detect the presence of a change in $\mathrm{K}$ by setting a threshold for the actual measured value of K. Next, MEC was determined based on the rate of change between each measured value and its pre-stimulus value. Figure 4 shows a histogram of the four MEC. MEC could be measured in 27 cases for K, 8 for HR, 21 for BP, and 25 for PPG. The frequency peaks were generally in the vicinity of 40-50 mA, except for $H R$, where MEC was very often outside the measurement range.

Then, in addition to the MEC of each parameter determined above, $K_{R 80}$, the rate of change in $K$ at the maximum current value $(80 \mathrm{~mA})$, was calculated. A scatter plot of each MEC and $K_{R 80}$ with $R C_{B P}$, respectively, is depicted in Figure 5. Pearson's correlation for each $M E C$ and $R O C_{B P}$ is $-0.723\left(M C_{K}\right)$, $-0.067\left(\mathrm{MEC}_{\mathrm{HR}}\right),-0.565\left(\mathrm{MEC}_{\mathrm{BP}}\right),-0.711$ ( $\left.\mathrm{MEC}_{\mathrm{PPG}}\right)(\mathrm{P}<0.001, \mathrm{P}=0.87, \mathrm{P}=0.009$, and $\mathrm{P}<0.001$, respectively). $\mathrm{MEC}_{\mathrm{K}}, \mathrm{MEC}_{\mathrm{BP}}$, and $M E \mathrm{C}_{\mathrm{PPG}}$ were shown to be significantly correlated with $\mathrm{ROC}_{\mathrm{BP}}$. Three cases for $\mathrm{MEC}_{K}, 9$ cases for $\mathrm{MEC}_{\mathrm{BP}}$, and 5 cases for $\mathrm{MEC}_{\mathrm{PPG}}$ were classified as out of the measurement 
range. The $\mathrm{ROC}_{\mathrm{BP}}$ of these out-of-range cases were $2.6 \%$ (-3.7 to 3.3$), 14.4 \%$ (2.6 to 23.3$)$, and $3.3 \%$ (-3.7 to 15.4 ), respectively.

Among the out-of-measurement range groups, there was no statistically significant difference in the $\mathrm{ROC}_{\mathrm{BP}}$ of $\mathrm{MEC}_{\mathrm{K}}$ and MEC $\mathrm{PPG}_{\mathrm{P}}$ measurement range groups. However, out-of-range cases of MEC $\mathrm{PPG}_{\mathrm{Pad}}$ a large degree of variability and included cases with large $\mathrm{ROC}_{\mathrm{BP}}$. Therefore, we determined that $\mathrm{MEC}_{\mathrm{K}}$ was the index with the highest degree of correlation with $\mathrm{ROC}_{\mathrm{BP}}$ among $\mathrm{MEC}_{\mathrm{K}}, \mathrm{MEC}_{\mathrm{HR}}, \mathrm{MEC}_{\mathrm{BP}}$, and $\mathrm{MEC} \mathrm{CPFG}_{\mathrm{P}}$.

We then used $\mathrm{MEC}_{\mathrm{K}}$ and $\mathrm{K}_{\mathrm{R} 80}$ to predict $\mathrm{ROC}_{\mathrm{BP}}$. First, for $\mathrm{MEC}_{\mathrm{K}}$, a first-order regression equation was applied after outlier treatment in the scatter plot in Figure $5 \mathrm{a}$, as follows:

Predicted ROC $\mathrm{BP}(\%) \otimes-0.28 \times \mathrm{MEC}_{K}(\mathrm{~mA})+31.26(r=0.723, \mathrm{P}<0.001),(4)$

Where the mean value of $\mathrm{ROC}_{\mathrm{BP}}$ in cases outside the $\mathrm{MEC}_{\mathrm{K}}$ measurement range was $0.7 \%$. This rate of change was substituted into the temporal regression equation, which yielded a value of $107(\mathrm{~mA})$. This value was used as a provisional $M E C_{K}$ value for cases outside the $M C_{K}$ measurement range. Next, for $\mathrm{K}_{\mathrm{R} 80}$, the first-order regression equation after outlier treatment in the scatter plot in Figure 5e was applied, as follows:

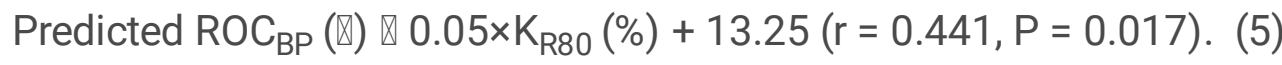

The above prediction equation was used to calculate the predicted $\mathrm{ROC}_{\mathrm{BP}}$. The results of the BlandAltman plot of measured and predicted values are shown in Figure 6. The difference between measured and predicted $\mathrm{ROC}_{\mathrm{BP}}$ is shown as the vertical axis and the mean as the horizontal axis. For $\mathrm{MEC}_{\mathrm{K}}$, fixed bias was small $(-0.17 \%)$, and the Pearson correlation coefficient in the scatter plot was $R=0.322(P=$ 0.088 ), and thus, no significant proportional bias was found. In contrast, for $\mathrm{K}_{\mathrm{R} 80}$, the fixed bias was small (-0.01\%); however, the Pearson correlation coefficient of the scatter plot contained significant proportional bias $(\mathrm{R}=0.714, \mathrm{P}<0.001)$.

\section{Discussion}

Although opioids are essential drugs for general anesthesia, inappropriate administration may lead to unpleasant effects on patients. Estimation of appropriate dosage is difficult because of large individual differences in opioid sensitivity. In this study, a prediction formula using $\mathrm{MEC}_{\mathrm{K}}$ as an indicator was able to predict $\mathrm{ROC}_{\mathrm{BP}}$ with high accuracy.

$M_{K} C_{K}$ is the threshold for the appearance of a sympathetic response when blood concentrations of opioids are $2 \mathrm{ng} / \mathrm{ml}$ and can be regarded as an intrinsic value that indicates individual opioid sensitivity. In other words, for patients with a low $\mathrm{MEC}_{\mathrm{K}}$, increasing the dose of opioids prior to skin incision can reduce circulatory fluctuations. The relationship between the $\mathrm{MEC}_{\mathrm{K}}$ and $\mathrm{ROC}_{\mathrm{BP}}$ at different opioid 
concentrations remains to be determined; however, the present results indicate that the $\mathrm{MEC}_{\mathrm{K}}$ may be a good indicator of opioid sensitivity.

$M E C_{K}$ and $K_{R 80}$

$\mathrm{MEC}_{\mathrm{K}}$ correlated better with $\mathrm{ROC}_{\mathrm{BP}}$ than $\mathrm{K}_{\mathrm{R} 80}$ (Figure 5) and precision was also small (Figure 6). This implies that $\mathrm{MEC}_{\mathrm{K}}$ was more accurate when predicting $\mathrm{ROC}_{\mathrm{BP}}$ than $\mathrm{K}_{\mathrm{R} 80}$. We previously reported that the rate of change in $\mathrm{K}$ with constant pain was inversely related to opioid concentration. However, there were large individual differences in the relationship between opioid concentration and the rate of change in $\mathrm{K}$ [9]. This may be due to individual differences in sympathetic response to noxious stimuli. When the primary afferent sensory nerve was depolarized by nociceptive stimulation, the signal was transmitted to the hypothalamus by the depolarization of the secondary afferent sensory nerve. A nociceptive signal input to the hypothalamus was transmitted to the sympathetic nerve center, and an efferent signal caused an autonomic nervous reaction such as blood vessel contraction, blood pressure increase, and heart rate change. Opioids suppress autonomic nervous responses by various actions such as transmitter release of primary afferent sensory nerves, suppression of depolarization of secondary afferent sensory nerves, and activation of the descending pain inhibitory system by disinhibition of the hypothalamus. The index of the response strength of $\mathrm{K}$ itself, such as $\mathrm{K}_{\mathrm{R} 80}$, measures the strength of the response when the noxious stimulus is transmitted to the sympathetic nerve center via the afferent and further to the peripheral effector through the centrifugal tract. Naturally, the presence or absence of afferent stimuli alters $\mathrm{K}_{\mathrm{R} 80}$; however, at the same time differences in the responsiveness of the effector also alter $\mathrm{K}_{\mathrm{R} 80}$. In contrast, $\mathrm{MEC}_{\mathrm{K}}$ is independent of effector responsiveness because it measures the presence or absence of sympathetic responses to nociceptive stimuli. Therefore, $\mathrm{MEC}_{\mathrm{K}}$ could predict blood pressure fluctuations associated with skin incision more accurately than the $\mathrm{K}_{\mathrm{R} 80}$.

\section{Correlation with $R_{O C} C_{B P}$ during skin incision}

$M C_{K}$ and $M E C_{P P G}$ correlated better with $R_{B P}$ versus $M E C_{H R}$ and $M E C_{B P}$, which suggests that $M E C_{K}$ and $\mathrm{MEC}_{\mathrm{PPG}}$ may capture sympathetic responses to nociceptive stimuli more accurately. In contrast, the number of cases outside the measurement range of $\mathrm{MEC}_{\mathrm{PPG}}$ was higher than that of the $\mathrm{MEC}_{\mathrm{K}}$ ( 6 versus 3 for $\mathrm{MEC}_{\mathrm{PPG}}$ and $\mathrm{MEC}_{\mathrm{K}}$, respectively), and $\mathrm{ROC}_{\mathrm{BP}}$ variability of $\mathrm{MEC}_{\mathrm{PPG}}$ was also higher. This may be because PPG amplitude reflects changes in blood flow and is influenced by multiple parameters such as cardiac output and vascular tone. The PPG amplitude was reduced, probably by vasoconstriction with nociceptive stimuli; however, it may have been increased despite nociceptive stimuli as a consequence of an augmentation factor that was a result of increased cardiac output that occurred at the same time [12].

In contrast, $\mathrm{K}$ was not affected by blood flow changes due to changes in cardiac output because it measured vascular compliance. This means that in cases where PPG cannot detect or shows excessive changes, $\mathrm{K}$ can accurately detect the presence or absence of a sympathetic response. For these reasons, we believe that the $\mathrm{MEC}_{\mathrm{K}}$ was the most accurate measure of MEC. 


\section{Limitations}

To determine if $\mathrm{MEC}_{\mathrm{K}}$ can be an indicator of opioid sensitivity, findings produced while adjusting the opioid dose based on $\mathrm{MEC}_{\mathrm{K}}$ must be compared with findings determined using a constant opioid dose. A study to further evaluate this is in the planning stage.

Remifentanil concentrations were examined solely at $2 \mathrm{ng} / \mathrm{ml}$. Changes in MEC and $\mathrm{ROC}_{\mathrm{BP}}$ at other concentrations require additional study. However, at $2 \mathrm{ng} / \mathrm{ml}, \mathrm{MEC}$ varied appropriately between 10-80 $\mathrm{mA}$. If the concentration of remifentanil was greater than $2 \mathrm{ng} / \mathrm{ml}$, the number of cases outside the measurement range was expected to increase. In contrast, when tested at concentrations less than 2 $\mathrm{ng} / \mathrm{ml}, \mathrm{MEC}$ values below $10 \mathrm{~mA}$ may have been uniformly regarded as MEC values of $10 \mathrm{~mA}$. These facts suggest that the concentration of $2 \mathrm{ng} / \mathrm{ml}$ used in this study was reasonable regarding the amount of opioids needed to inhibit tetanus stimulation.

Sympathetic response also occurs in muscle contraction induced by tetanus stimulation. Therefore, tetanus stimulation is not considered to be a pure $A \delta$ or $C$ fiber stimulus. However, Funcke et al. stated that the sympathetic responses to tetanus and intradermal electrical stimulation might be considered as a similar stimulus [13]. In this study, the tetanus stimulus was highly specific to $A \delta$ and $C$ fibers in the inhibition of muscle contraction due to the administration of muscle relaxants.

\section{Conclusions}

Compared with $\mathrm{K}_{\mathrm{R} 80}, \mathrm{MEC}_{\mathrm{HR}}, \mathrm{MEC}_{\mathrm{BP}}$, and $\mathrm{MEC} \mathrm{PPG}_{\mathrm{PP}}, \mathrm{MEC}_{\mathrm{K}}$ correlated best with blood pressure changes during skin incisions. The $\mathrm{MEC}_{\mathrm{K}}$-indexed prediction equation accurately predicted the increase in blood pressure that occurred during skin incision at a remifentanil effect-site concentration of $2 \mathrm{ng} / \mathrm{ml}$. $\mathrm{MEC}_{\mathrm{K}}$ was shown to be a potentially more accurate indicator of opioid sensitivity than the rate of change in $\mathrm{K}$.

\section{Declarations}

Funding: Institutional and/or departmental

Competing interests: The authors have no competing interests to declare.

Author contributions: SK helped design the study, analyze, and interpret the data, and draft and approved the final version of the manuscript. RN helped conceive and design the study, analyze, and interpret the data, draft the manuscript, and approved the final version. NS helped design the study, analyze, and interpret data and approved the final manuscript. TK was involved in recruitment and data collection and approved the final manuscript. HM assisted with recruitment and data collection and approved the final manuscript. SN assisted with recruitment and data collection and approved the final manuscript. AM assisted with recruitment and data collection and approved the final manuscript. MK assisted with the initial design of the study and approved the final manuscript. $\mathrm{HH}$ assisted with the initial design of the 
study and approved the final manuscript. TT assisted with the initial design of the study and approved the final manuscript. YMT helped design the study, interpreted data, and approved the final manuscript.

\section{References}

1. Luginbühl, M., Reichlin, F., Sigurdsson, G., Zbinden, A., \& Peterson-Felix, S. Prediction of the haemodynamic response to tracheal intubation: comparison of laser-Doppler skin vasomotor reflex and pulse wave reflex. Br. J. Anaesth. 89, 389-397 (2002).

2. Allen, J. Photoplethysmography and its application in clinical physiological measurement. Physiol. Meas. 28, R1-R39 (2007).

3. Huiku, M., et al. Assessment of surgical stress during general anaesthesia. Br. J. Anaesth. 98, 447455 (2007).

4. Boselli, E., Bouvet, L., Begou, G., Torkmani, S., \& Allaouchiche, B. Prediction of hemodynamic reactivity during total intravenous anesthesia for suspension laryngoscopy using analgesia/nociception index (ANI): A prospective observational study. Minerva Anestesiol. 81, 288297 (2015).

5. Sakane, A., Tsuji, T., Tanaka, Y., Saeki, N., \& Kawamoto, M. Monitoring of vascular condition using plethysmogram. SICE. 40, 1236-1242 (2004).

6. Matsubara, H., et al. Quantitative evaluation of pain during electrocutaneous stimulation using a loglinearized peripheral arterial viscoelastic model. Sci. Rep. 8, 1-9 (2018).

7. Saeki, N., Nakamura, R., Kawamoto, M., Tsuji, T. \& Shiba, K. Vascular impedance and pulse wave velosity during sympathetic blockade. Anesthesiology. 107, A1257 (2007).

8. Siba, K., Toshio, T., Hiroshi, H., \& Masashi, K. Arterial mechanical impedance is a sensitive stress response monitor during general anesthesia. Hiroshima J. Med. Sci. 58, 75-82 (2009).

9. Yanabe, K., et al. A new arterial mechanical property indicator reflecting differences in invasive stimulus intensity induced by alteration of remifentanil concentration during laryngoscopy. Minerva Anestesiol. 84, 311-318 (2018).

10. Sukhdorj, E., et al. Response of arterial mechanical impedance to different concentrations of remifentanil during abdominal laparoscopic colectomy. Hiroshima J. Med. Sci. 67, 55-61 (2018).

11. Minto, C. F., Schnider, T. W., \& Shafer, S. L. Pharmacokinetics and pharmacodynamics of remifentanil. II. Model application. Anesthesiology. 86, 24-33 (1997).

12. Korhonen, I., \& Yli-Hankala, A. Photopletysmography and nociception, Acta Anaesthesiol. Scand. 53, 975-985 (2009).

13. Funcke, S., et al. Validation of innovative techniques for monitoring nociception during general anesthesia: a clinical study using tetanic and intracutaneous electrical stimulation. Anesthesiology. 127, 272-283 (2017).

\section{Table}


Table 1 Patient characteristics

\begin{tabular}{|ll|}
\hline Male/Female & $15 / 15$ \\
\hline Age $($ years $)$ & $62 \pm 13(35-80)$ \\
\hline Height $(\mathrm{cm})$ & $161.7 \pm 9.7(143.3-177.8)$ \\
\hline Weight $(\mathrm{kg})$ & $56.3 \pm 13.3(36.9-99.6)$ \\
\hline BMI $\left(\mathrm{kg} / \mathrm{m}^{2}\right)$ & $21.6 \pm 4.8(12.5-39.9)$ \\
\hline Upper/lower abdomen & $13 / 17$ \\
\hline sBP pre-incision $(\mathrm{mmHg})$ & $77.8 \pm 13.3(56.0-108.0)$ \\
\hline sBP post-incision $(\mathrm{mmHg})$ & $91.7 \pm 16.1(57.4-126.0)$ \\
\hline Rate of change in sBP before and after incision $(\%)$ & $18.3 \pm 11.8(-3.7-38.4)$ \\
\hline
\end{tabular}

BMI: body mass index, sBP: systolic blood pressure

Results are presented as mean $\pm S D$ (minimum-maximum).

Figures 


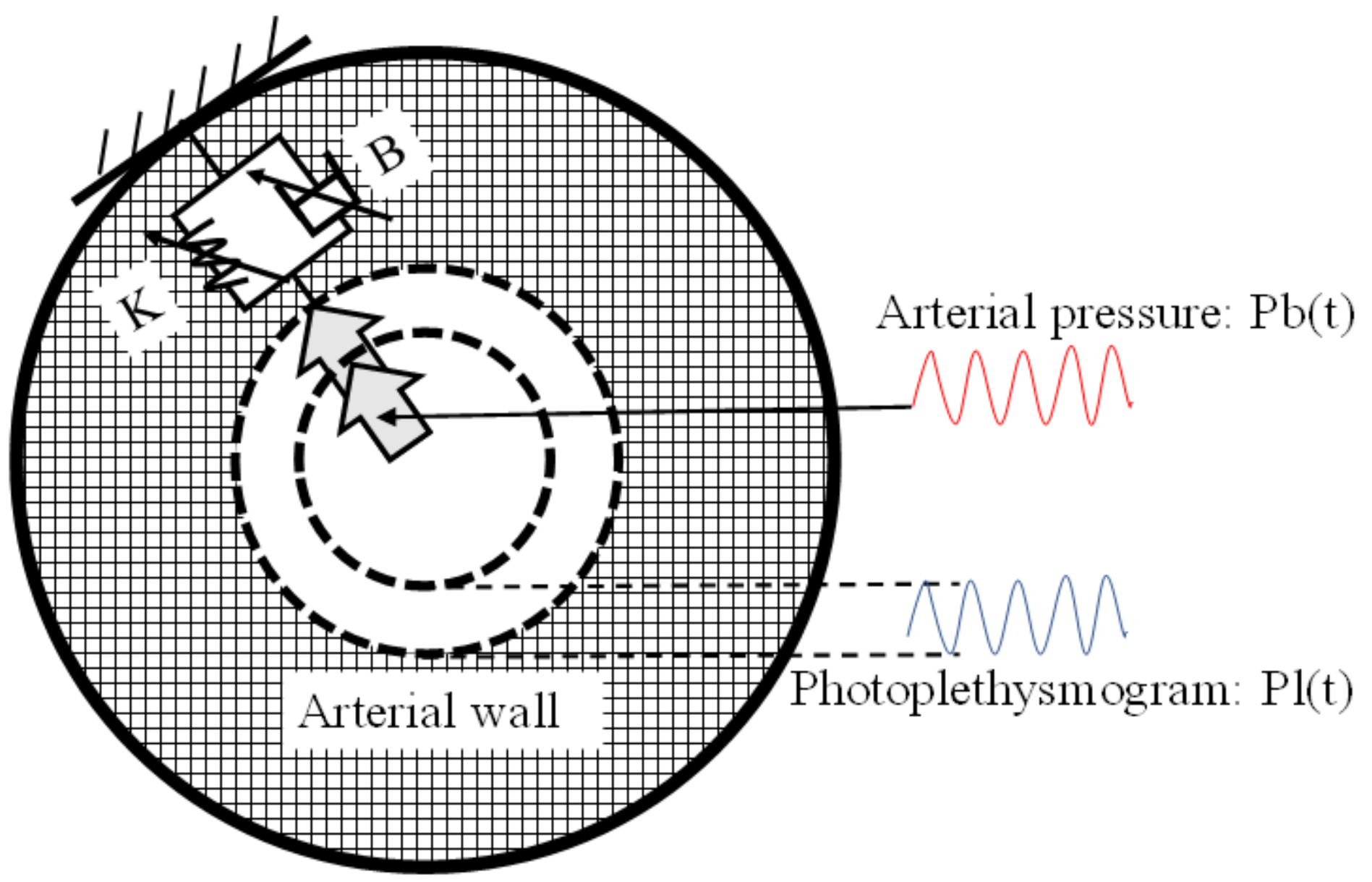

Figure 1

Schematic diagram of the mechanical impedance model. The model used in this study consists of a parallel arrangement of springs and dampers across the diameter of the arterial wall. When a force (blood pressure) is applied across the diameter of the artery, the arterial wall is dampened by springs and dampers that represent the rigidity and viscosity of the arterial wall itself. The behavior of the arterial wall after damping is observed as a change in the photoplethysmogram waveform. 


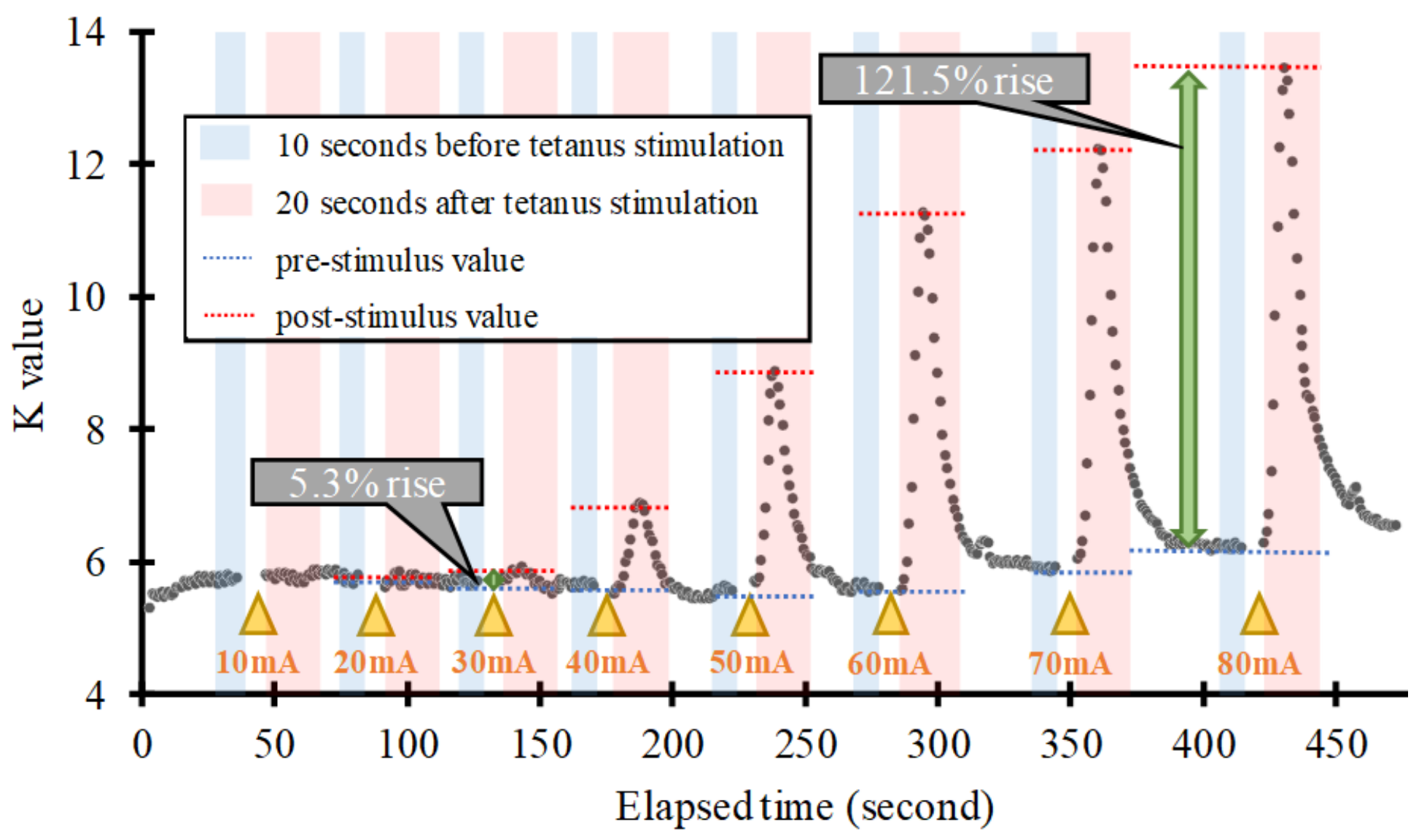

Figure 2

Schema describing minimum evoked current (MEC) measurement in the study. An example of variation in vascular stiffness value $(K)$ during tetanus stimulation. The predicted effect-site concentration of remifentanil was maintained at $2 \mathrm{ng} / \mathrm{ml}$ throughout the study. In the figure, $\triangle$ indicates the timing of tetanus stimulation, which was incrementally increased by $10 \mathrm{~mA}$. The rate of $\mathrm{K}$ change increased with increasing stimulus intensity. In this case, the first time the rate change of $K$ exceeded $5 \%$ was after 30 $\mathrm{mA}$ stimulation (MECK $=30 \mathrm{~mA}$ ). The rate of change in $\mathrm{K}$ at $80 \mathrm{~mA}$ is $121.5 \%$, which is $\mathrm{KR} 80$. 

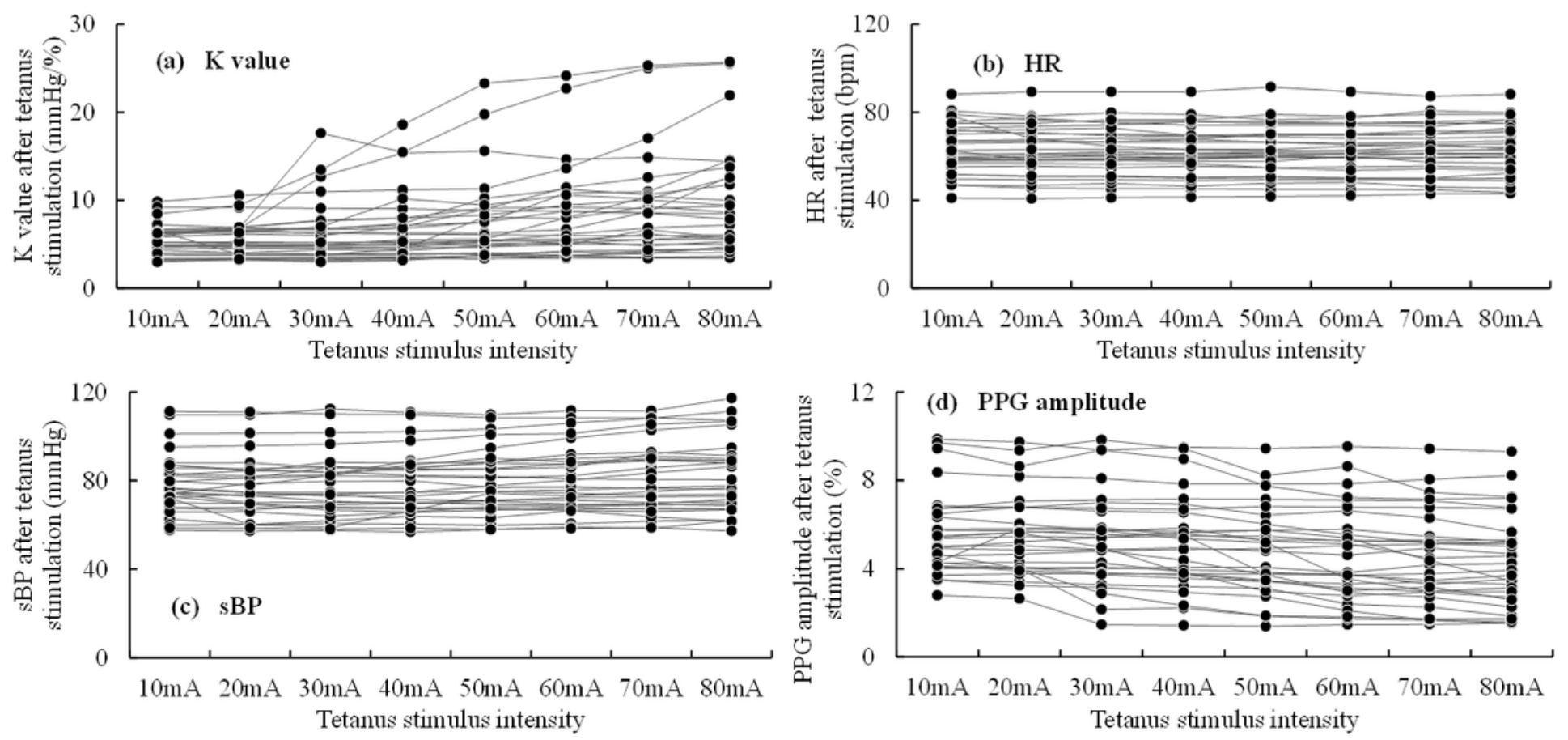

Figure 3

Vascular stiffness value (K), systolic blood pressure (sBP), heart rate (HR), and photoplethysmography amplitude (PPG) after tetanus stimulation at each intensity. There were many cases in which the value of $\mathrm{K}$ increased with increasing stimulus intensity. However, when the stimulus intensity was large, individual differences in the $\mathrm{K}$ change tended to be large. Values of PPG amplitude tended to decrease with increasing stimulus intensity. In many cases, there was no obvious change in HR even when the stimulus intensity was increased. 
$\mathrm{MECK}$

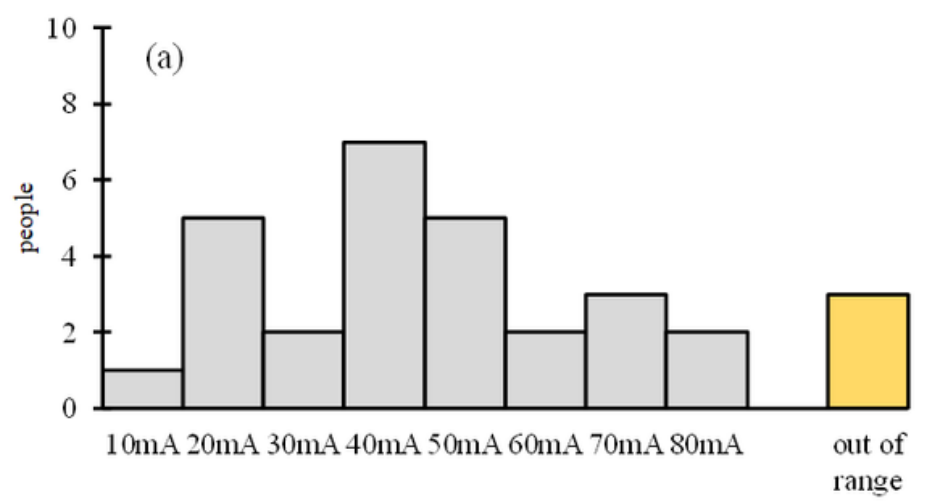

$M \mathbb{E} \mathrm{C}_{\mathrm{BP}}$

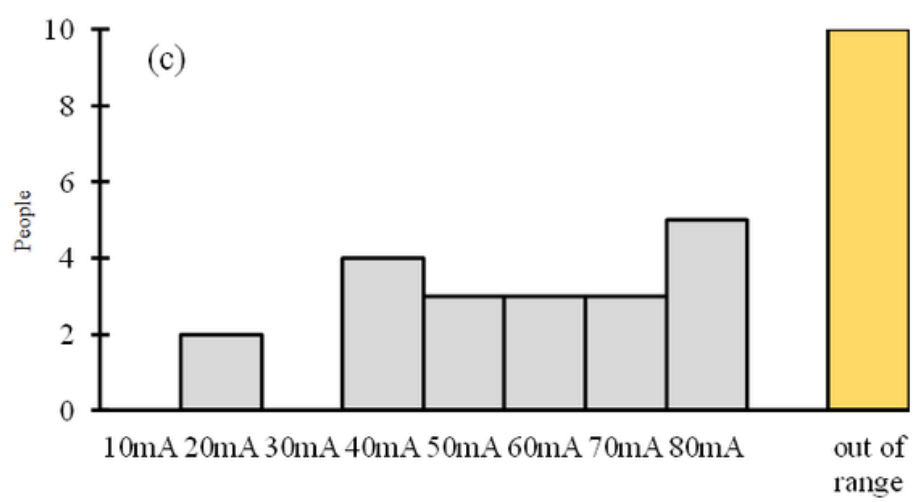

MECHR

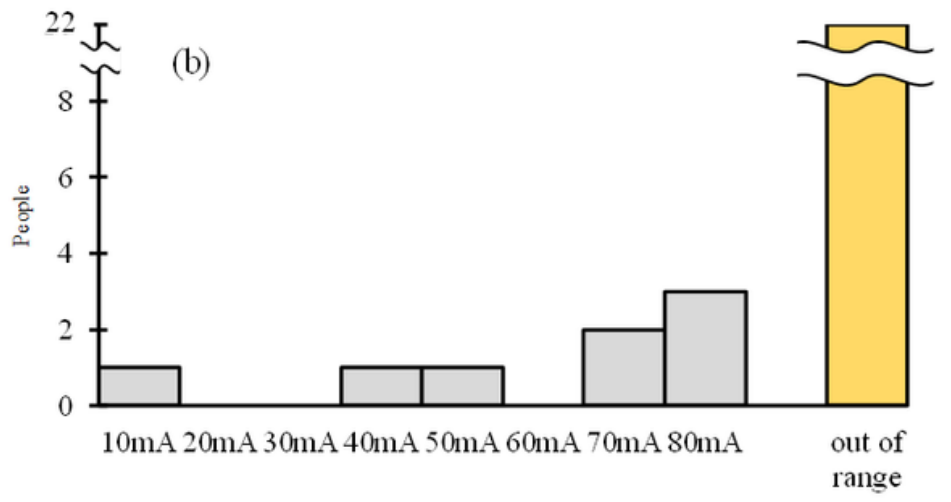

MECPPG

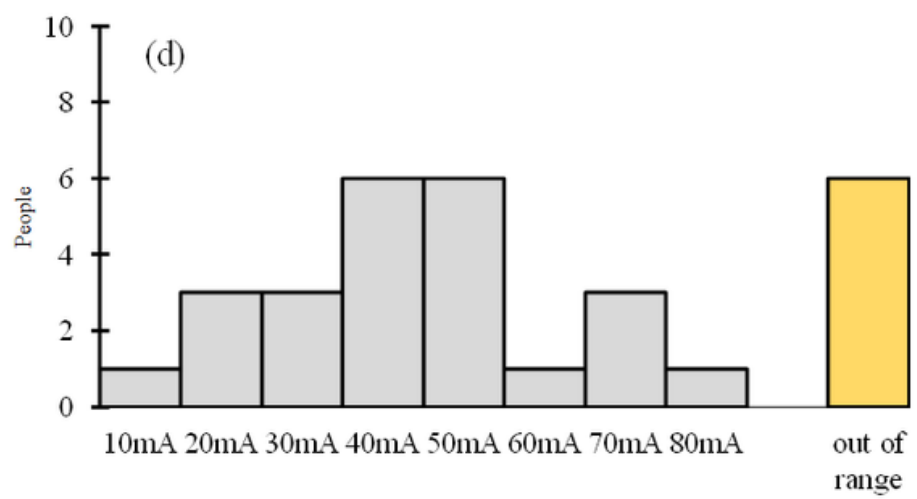

Figure 4

Histogram of each minimum evoked current (MEC). The distributions of MEC, vascular stiffness value $(\mathrm{K})$, systolic blood pressure (BP), heart rate (HR), and photoplethysmography amplitude (PPG) are shown. MEC represents the minimum stimulus current intensity at which a change of more than $5 \%$ in the parameter appears for tetanus stimuli given in $10 \mathrm{~mA}$ increments. Except for HR, which was very often outside the measurement range, frequency peaks were generally around 40-50 mA. 

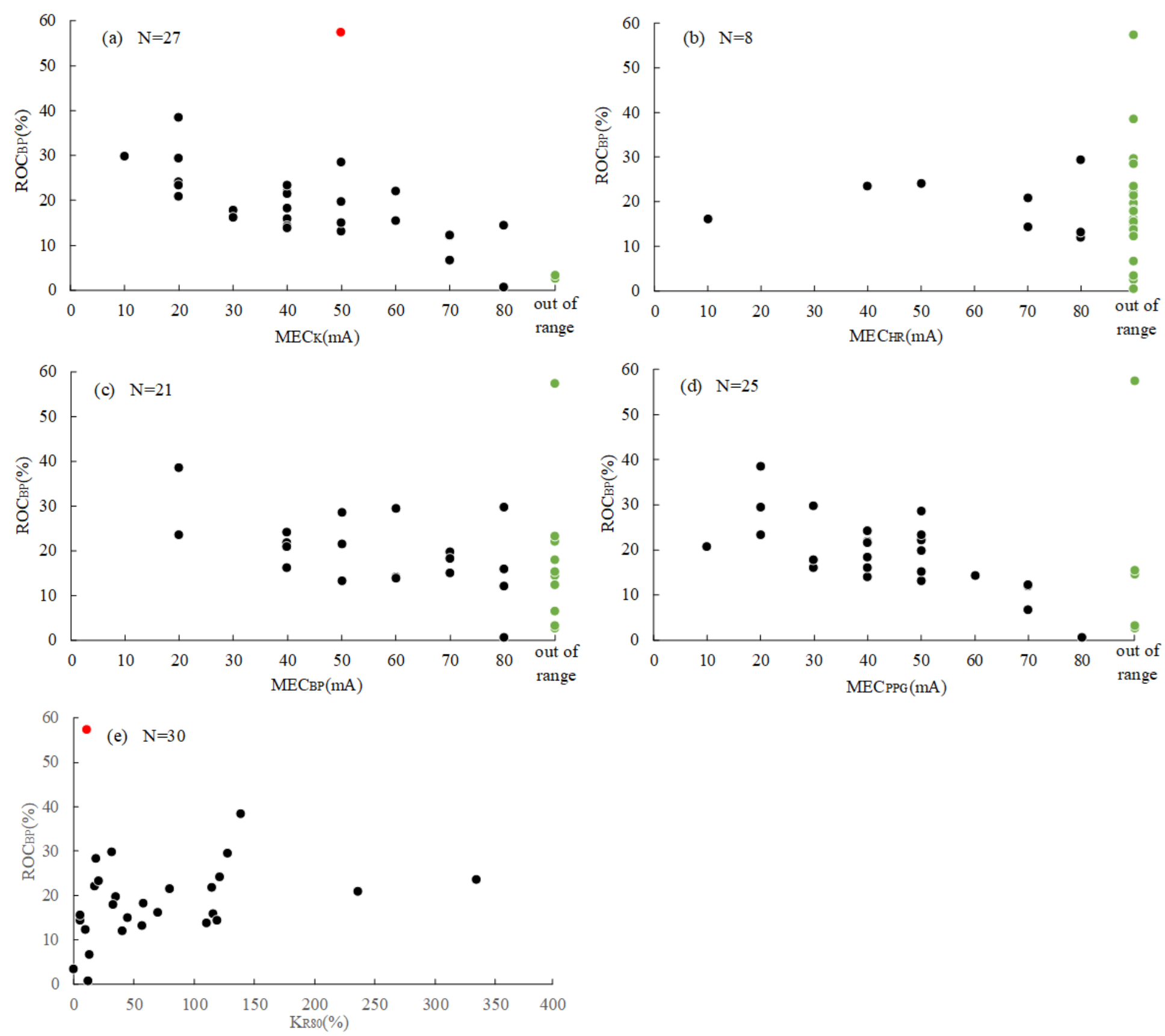

Figure 5

Relationship between minimum evoked current (MEC)+ rate of change in $\mathrm{K}$ for $80 \mathrm{~mA}$ tetanus stimulation (KR80) and rate of change of systolic blood pressure (ROCBP). A scatter plot of each minimum evoked current (MEC), vascular stiffness value (K), systolic blood pressure (sBP), heart rate (HR), photoplethysmography amplitude (PPG), and rate of change of systolic blood pressure (ROCBP) is shown. Out-of-range cases are shown using green points, and Smirnoff-Grubbs test outlier points are shown in red. A downward rightward trend is observed for MECK, MECBP, and MECPPG, and an upward rightward trend is observed for KR80. 


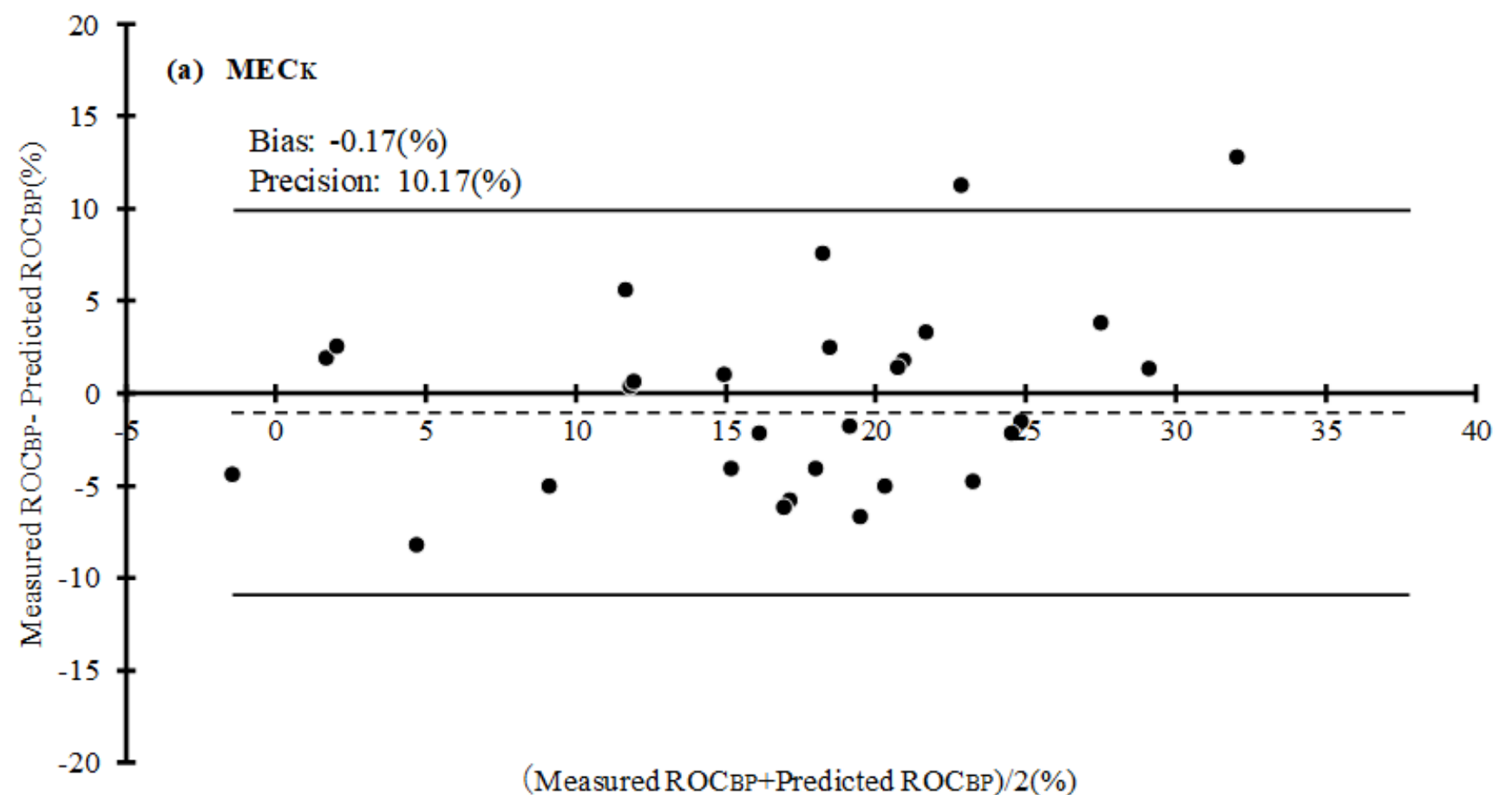

(Measured ROCBP+Predicted ROCBP)/2(\%)

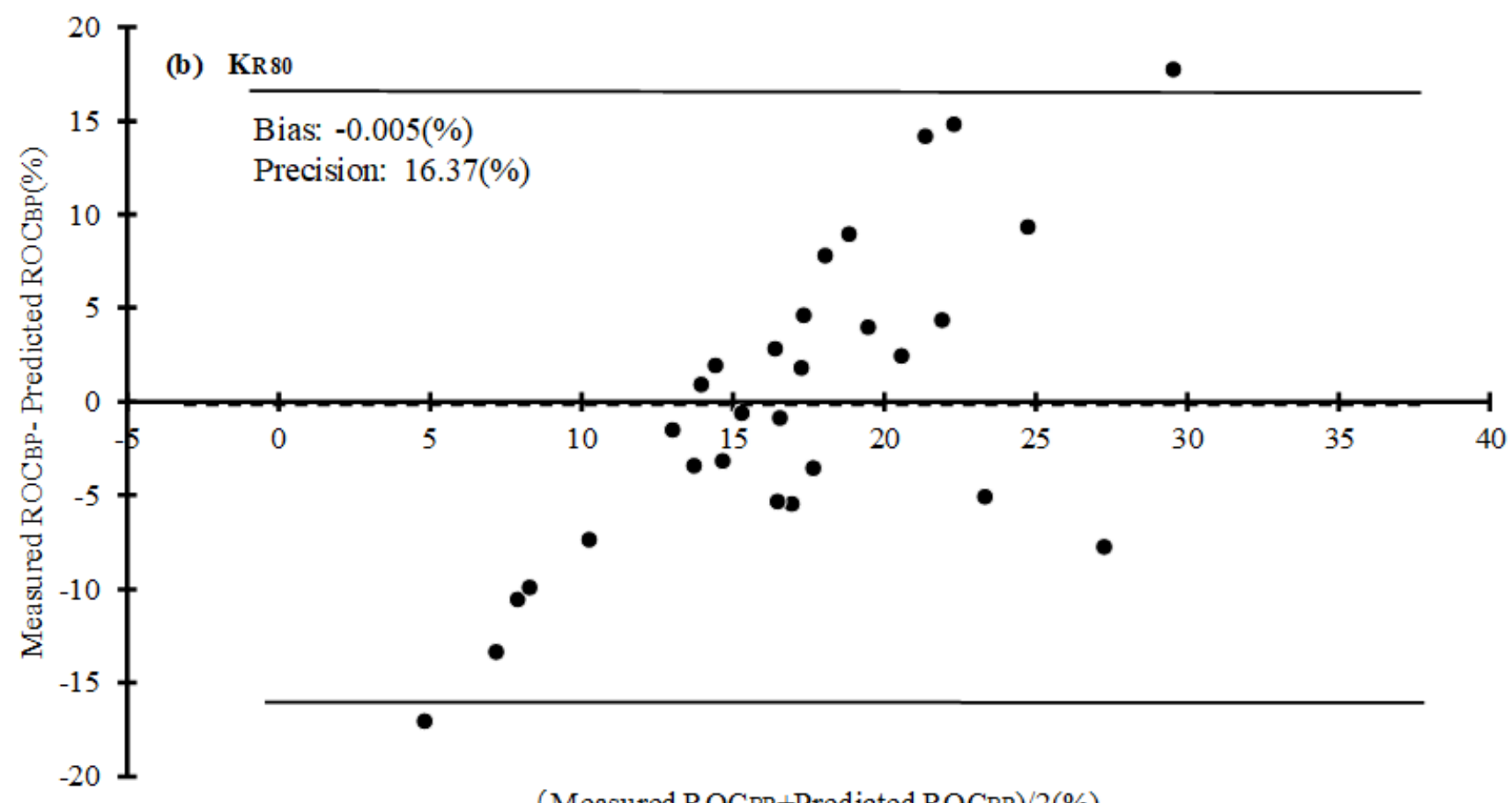

(Measured ROCBP+Predicted ROCBP)/2(\%)

\section{Figure 6}

Comparison of the accuracy of ROCBP predictions using MECK (a) and KR80 (b). The error associated with predicted and measured rates of change of systolic blood pressure (ROCBP) estimated by MECK and KR80, respectively, are shown. The fixed bias was determined to be small since the data used to create the prediction equation, and data used for validation were the same. The proportional error of MECK was 
small. KR80 displayed a definite proportional error, and its prediction performance was determined to be low. 